\title{
Anti-Haecceitism and Fundamentality
}

\author{
Maria Scarpati ${ }^{1}$
}

Received: 29 August 2020 / Accepted: 16 November 2021

(c) The Author(s) 2021

\begin{abstract}
Is everything about reality either qualitative or somehow determined by the qualitative character of reality itself? Metaphysical anti-Haecceitism is often taken to be the claim that this is the case, and to entail that reality is fundamentally qualitative. In this paper, I (1) argue against the idea that metaphysical anti-Haecceitism should be characterized in such terms, and (2) defend a novel way to phrase such a view. This will be done by taking the main arguments for anti-Haecceitism as a guide and by singling out the proper commitments of a view that satisfies the desideratum set forth by such arguments. The phrasing I shall offer will help tell the issue of antiHaecceitism apart from some concerns about fundamentality that, I will contend, are orthogonal to it - among them, the question whether there is fundamentality at all.
\end{abstract}

This paper deals with the disagreement between metaphysical Haecceitism and metaphysical anti-Haecceitism. More precisely, it attempts to properly define such a disagreement and to clearly tell it apart from some other disputes with which it is easily conflated.

Importantly, the debate I am engaging is not the one between the doctrines that have been called modal Haecceitism and modal anti-Haecceitism-i.e., the one as to whether maximal possibilities ever differ in what they represent de re while not differing in their qualitative character at all. ${ }^{1}$ It is, rather, a time-honoured debate that was brought to the attention of contemporary metaphysics by Robert M. Adams. Adams's treatment of the topic starts with the following query ('Adams's question', henceforth):

\footnotetext{
1 The issue of modal Haecceitism is thus stated in Lewis (1986): 220-247 and based on Kaplan (1975). For modal Haecceitism, see Cowling (2016) and literature therein. For a first explicit attempt to distinguish metaphysical Haecceitism from modal Haecceitism, see Fine (2005): 19-39. More on this in due course.
}

Maria Scarpati

maria.scarpati@unine.ch

1 Philosophy Department, University of Neuchâtel, Espace Tilo-Frey 1, 2000 Neuchâtel, Switzerland 
Is the world — and are all possible worlds — constituted by purely qualitative facts or does thisness hold a place beside suchness as a fundamental feature of reality ${ }^{2}$

To a first approximation, metaphysical anti-Haecceitism might be thought as the claim that the former is the case-i.e., that reality is "constituted by purely qualitative facts". ${ }^{3}$ Haecceitism, ${ }^{4}$ by contrast, might roughly be viewed as holding that the latter is true instead - that "thisness hold[s] a place beside suchness as a fundamental feature of reality". 5

According to the analysis Adams provided, anti-Haecceitism "stands or falls (...) with a certain doctrine of the Identity of Indiscernibles". ${ }^{6}$ This is because-so Adams has it - to be an anti-Haecceitist is to hold that no entity has "primitive, nonqualitative thisness". ${ }^{7}$ Having a certain thisness simply is, for Adams, to be a certain entity in particular. Thus, anti-Haecceitists would hold that every entity is the very entity it is inasmuch as it is qualitatively a certain way-i.e., that every entity is individuated by its qualitative profile. And the latter claim entails, in turn, that every entity fulfils a strong version of the Identity of Indiscernibles (PII) - that is, that no two entities can be qualitatively just alike. ${ }^{8}$

Adams's approach to the topic, however, seems to have fallen into disuse at least since Dasgupta $(2009,2014,2017)$ defended a new phrasing of anti-Haecceitism and its rival view-Haecceitism (in Dasgupta's terms, of 'Qualitativism' and 'Individualism'). Dasgupta's ultimate aim in the mentioned papers is to characterize and defend a metaphysics that is anti-Haecceitist about material entities. According to his approach, the anti-Haecceitist can avoid focusing on such material entities and their thisnesses-hence, on issues concerning how material entities are individuated-thus foregoing the commitment to PII. The idea is that one can sidestep the material entities altogether and still make sure that one's metaphysics is anti-Haecceitist about them as long as it holds that all fundamental (i.e., roughly, ungrounded) facts are purely qualitative. ${ }^{9}$ Dasgupta makes this point while presenting the bundle theory of universals as a viable way to be an anti-Haecceitist. Viable but not satisfactory, he thinks, exactly because it commits one to PII and thus to excluding the possibility of a world where, as in Black (1952), nothing exists but two mutually

\footnotetext{
2 Adams (1979): 5.

3 Ibid.

4 By 'Haecceitism' and 'anti-Haecceitism', I will henceforth mean the two metaphysical doctrinesunless otherwise specified.

5 Adams (1979): 5.

6 Ibid:: 11.

7 Ibid:: 5 .

8 I will get back to this point in due course.

9 Under Dasgupta's approach, the fundamental facts cannot literally be identified with the ungrounded ones insofar as Dasgupta (2014) defends the idea that facts $F^{\prime}, F^{\prime},, \ldots, F^{n}$ at least "derivatively ground" (ibid.: 6) a fact $F$ iff $F^{\prime}, F^{\prime},, \ldots, F^{n}$ ground a plurality of facts that includes $F$ together with some further facts-even if they do not ground $F$ on its own. The fundamental facts would then be those facts that are not even derivatively grounded in other facts.
} 
indiscernible individuals. The world in question, says Dasgupta, can however "be characterized in purely general terms by the following sentence: ${ }^{10}$

$$
\text { (A) } \exists x \exists y(\mathrm{~F} x \& \mathrm{~F} y \& x \neq y)^{\prime \prime} \text {. }
$$

Contra the bundle theorist, Dasgupta then suggests that the anti-Haecceitist should not "ask how to construct individuals out of more basic entities". ${ }^{11}$ She should "simply ask for an account of the fundamental structure of the world that dispenses with primitive individuals but which allows us to make sense of the whole array of possible general facts, including that expressed by (A), and which therefore does not imply [PII]". 12

That the disagreement between Haecceitism and anti-Haecceitism can be effectively phrased in terms of grounding and fundamentality is then crucial for Dasgupta's project.

Since Dasgupta's approach was set forth, anti-Haecceitism has widely been viewed as the claim that all fundamental facts are qualitative. ${ }^{13}$ In this paper, however, I aim to cast doubts on the idea that anti-Haecceitism can satisfyingly be phrased in terms of grounding and fundamentality. Contra Dasgupta, a phrasing of the present debate should indeed focus, I will contend, on entities, and on whether they_or at least some of them — are such that they might offend against what I will argue to be the rationale for anti-Haecceitism. An important merit of my characterization of anti-Haecceitism is precisely that adopting it allows us to keep the debate about such a view explicitly separated from some concerns about fundamentality that, I will argue, are orthogonal to it-among them, the question whether there is a fundamental level at all.

I will start by considering a phrasing of the debate that views anti-Haecceitism as the claim that reality is fundamentally qualitative while ignoring the idea that its rival view at least concerns the notion of thisness, hence simply taking Haecceitism to deny that reality is fundamentally qualitative (Sect. 1). Such a characterization has the surprising effect of qualifying every view that denies there to be fundamentality as a form of Haecceitism. I will point out that it should then be recognized to be flawed in case it turned out that some view can both (a.) maintain that there is no fundamentality, and (b.) satisfy the desideratum on metaphysics that the main arguments for anti-Haecceitism support. I will then introduce what I take to be the main families of arguments for anti-Haecceitism and single out the rationale they share and the desideratum such a rationale, if right, imposes (Sect. 2). The rationale, I will maintain, consists in the idea that what I will call 'haecceitistic differences' are for some relevant reason to be ruled out. But such an idea, as I will contend, can

\footnotetext{
10 Dasgupta (2009): 48. Dasgupta uses the terms 'general' and 'qualitative' as equivalent. $F$ would stand in (A) for the only universal instantiated by both individuals. I slightly modified the notation in (A) for reasons of overall consistency.

11 Ibid.

12 Ibid. For the notion of a primitive individual as employed by Dasgupta, see Sect. 2 below. Thanks to an anonymous referee for asking me to clarify this point.

13 See, in particular, Russell (2016, 2018), Bacon (2019), Sider (2020).
} 
be seconded in views that do not accept there to be fundamentality. I will offer, in particular, the example of an infinitist metaphysics in which haecceitistic differences cannot possibly arise (Sect. 3). I will then set forth a novel phrasing of anti-Haecceitism and show that it rightly classifies infinitist metaphysics as forms of anti-Haecceitism if they exclude the possibility of a haecceitistic difference. I will also discuss how this novel phrasing accommodates different intuitions about certain-allegedly haecceitistic_-worlds (Sect. 4).

\section{Must There Be Fundamentality for the Issue to Arise?}

Let us consider Adams's question again. If we take its face value as a guide to phrasing this debate and ignore the notion of thisness, it seems natural to simply regard anti-Haecceitism as the claim that reality is fundamentally qualitative.

For while prima facie Adams enquires whether all possible worlds are "constituted by purely qualitative facts" 14 simpliciter, he mentions that a negative answer to such a query would entail that thisness is a fundamental feature of reality. And this seems to imply that the notion of constitution he has in mind is one that tracks relative fundamentality. ${ }^{15}$ Of course, a stronger reading of Adams's question would suggest that the anti-Haecceitist takes all of reality — not only its fundamental level— to be qualitative. ${ }^{16}$ For several reasons, I take it that this would be too strong a claim to ascribe to the anti-Haecceitist. While a detailed enquiry into this point lies beyond my present aims, I will briefly get back to it in Sect. 4. More precisely, I will:

- Show that the anti-Haecceitist who means to hold that all of reality is qualitative can perfectly well express such a tenet in terms of the phrasing I will defend, and

- Argue that well-known arguments for anti-Haecceitism do not actually support that tenet, but only a weaker one.

The idea that taking the first horn of Adams's question is to maintain that all fundamental facts are qualitative is in any event worth addressing since recent, influential treatments of the topic crucially rest on it. As I said, this is not only the case for Dasgupta (2009, 2014, 2017), but also for Russell (2016, 2018), Bacon (2019), and Sider (2020). ${ }^{17}$

Now, what about Haecceitism? To stress once more: suppose we aimed to characterize the view by taking Adams's question as a guide and disregarding his hint to the fact that Haecceitism is, perhaps among other things, a claim about thisness. ${ }^{18}$

\footnotetext{
14 Adams (1979): 5.

15 For an endorsement of the idea that constitution is one of the relations that track relative fundamentality, see Bennett $(2011,2017)$.

16 The phrases 'qualitative' and 'purely qualitative' are to be taken as plainly synonymous here.

17 See also Babic and Cocco (2020).

18 As I said, after introducing the topic through Adams's question, Adams himself crucially shapes his analysis around the topic of thisness. According to that analysis, a Haecceitist holds that at least some things have primitive (i.e., non-qualitative) thisness. An anti-Haecceitist denies such a claim.
} 
A natural choice would then seem to take it that Haecceitism is simply the denial of anti-Haecceitism as defined above. It is — so the thought would go- the claim that it is not the case that reality is fundamentally qualitative.

A characterization of the two views would then go as follows.

ANTI-HAECCEITISM. Reality is fundamentally qualitative.

HAECCEITISM. It is not the case that reality is fundamentally qualitative.

Contra Adams's question and the approach inaugurated by Dasgupta - at least when taken at face value-the characterizations I suggest we consider do not make any appeal to facts. In particular, they do not assume that for reality to be fundamentally so-and-so is for it to be constituted by facts that are so-and-so and perhaps by other facts all of which are determined by some so-and-so ones. There is an important reason for this: I think that one need not allow facts in one's ontology in order to make claims concerning the fundamental character or reality. For instance, it has been argued that one can consistently characterize fundamentality in terms of grounding-more precisely, as ungroundedness, as what grounds everything else, or as both $-{ }^{19}$ while denying that true grounding statements ascribe a relation, and thus that there are entities related by a grounding relation in the first place. ${ }^{20}$ More importantly for my aims here, one can be either a Haecceitist or an anti-Haecceitist while denying that there are facts at all, as will be clear in the light of my phrasing of such views. $^{21}$

Anti-Haecceitism thus characterized is a conjunctive claim: it has it that (i.) there is a fundamental level of reality, in some sense, and that (ii.) such a fundamental level is qualitative.

Haecceitism, by contrast, is a disjunctive claim - the claim that either (i.*) there is no fundamental level of reality, or (ii.*) there is a fundamental level of reality in some sense, and it is not qualitative.

The tentative characterizations I am considering thus qualify every view that denies there to be a fundamental level of reality as a form of Haecceitism. Is this correct? I claim that it is certainly not if it turns out that one can both:

- Hold that there is no fundamental level of reality, and, consistently with this,

- Defend a metaphysics that satisfies the desideratum set forth by the typical arguments for anti-Haecceitism.

The next section will be devoted to singling out such a desideratum. Once that is done, I will make the case that the desideratum can be satisfied within views that

\footnotetext{
19 See Leuenberger (2020). Cf. also Raven (2016).

20 See Fine (2001) and Correia (2010).

21 Incidentally, Dasgupta seems to agree. In On the Plurality of Grounds he declares that his "official approach is to treat grounding as a sentential connective" and that his "talk of facts is just a convenient shorthand"; Dasgupta (2014): 4. Whether Dasgupta can indeed defend the sort of anti-Haecceitism he favors without ultimately committing to the existence of facts or not is a point that lies beyond the scope of the present paper. Thanks to an anonymous referee for having me clarify this point.
} 
deny there to be a fundamental level—hence, that the phrasings I am considering are wrong-headed.

\section{The Case for Anti-Haecceitism}

It might seem odd to consider the arguments for a claim — anti-Haecceitism—-that has not yet been duly characterized. I argue that the move is presently innocuous, though, inasmuch as the arguments that I will consider were presented in support of views that either explicitly take the first horn of Adams's question or were classified as doing so by Adams himself. Moreover, I think that looking at the typical arguments for anti-Haecceitism is exactly the right strategy towards providing a characterization of that view. For this ensures that the view thereby formulated is the one that matters in the light of its advocates' theoretical aims. ${ }^{22}$

While the arguments I will consider come from at least two diverse traditions-a broadly theistic one and a broadly empiricist one-the desideratum they set forth is one and the same. Such a desideratum is that what I will call haecceitistic differences be ruled out.

By a 'haecceitistic difference', I mean a case of a difference merely as concerns which entities are involved in particular situations. In other words:

Situation $S_{1}$ and situation $S_{2}$ differ haecceitistically iff $S_{1}$ and $S_{2}$ differ merely as concerns the identity of some of the entities they involve, respectively.

When I use the phrase 'situation', I do not mean to introduce any unfamiliar notion. Suppose, for instance, that Satchmo is playing the trumpet. Some think there exists then a fact that Satchmo is playing the trumpet; some that a state of affairs that Satchmo is playing the trumpet obtains; some that the proposition that Satchmo is playing the trumpet is true, and so on. But some hold that none of such readings is mandatory: all there is to say is that Satchmo is playing the trumpet. In this example, I would then speak of the situation that Satchmo is playing the trumpet in order to stay neutral as to whether this entails there being a fact, a state of affairs, a proposition, or else. ${ }^{23}$

Consider now a situation $S$ that Satchmo is playing the trumpet and a situation $S^{*}$ that is in every way like $S$ except that someone who is not Satchmo is playing the trumpet. $S$ and $S^{*}$ would differ haecceitistically in my sense. For they would differ merely as concerns the identity of an entity they involve, respectively ( $S$ involves Satchmo, while $S^{*}$ involves someone who is not Satchmo). Of course, the trumpet

\footnotetext{
22 Thanks to Shamik Dasgupta here.

23 Cf. Correia (2016), where the term 'situation' is used, roughly, as an unambiguously worldly counterpart of 'fact' - so that speaking of a situation described by a sentence $A$ is to speak of a going-on that is reported by $A$-ibid.: 104. There is a further important difference between Correia's notion of a 'situation' and the one commonly associated to the term 'fact': while the latter has in its extension only obtaining situations, the former has in its extension both obtaining and non-obtaining situations; see ibid. This, however, has no consequence for the present work. Thanks to an anonymous referee for seeking clarifications on this point, and to Fabrice Correia for discussion.
} 
player in $S^{*}$ would then be indiscernible from Satchmo. Otherwise, $S$ and $S^{*}$ would not differ merely as concerns the identity of some entities involved in them-they would also differ as concerns how some entities involved in them are. Consider, e.g., a situation $S^{* *}$ that is in every way like $S$ except that in $S^{* *}$ Miles Davis is playing the trumpet. $S$ and $S^{* *}$ do not differ haecceitistically (nor do $S^{*}$ and $S^{* *}$, for that matter): they differ as concerns the identity of an entity involved in them, but also in other respects. For instance, the trumpet player in $S$ is $1.68 \mathrm{~m}$ tall (as is the one in $\left.S^{*}\right)$, while the trumpet player in $S^{* *}$ is $1.69 \mathrm{~m}$ tall. ${ }^{24}$

(This is not to say that those things that can give rise to haecceitistic differencesif such things there be-have haecceities, where, for any thing $a, a$ 's haecceity is the property of being identical to $a$ and $a$ is the very thing it is by virtue of having such a property. Suppose $S$ and $S^{*}$ are both possible, and thus differ haecceitistically. One need not posit a haecceity of Satchmo and a haecceity of the trumpet player in $S^{*}$ in order to tell the two musicians apart from one another. One can simply hold that each musician is primitively individuated, and that $S$ and $S^{*}$ are mutually distinct in virtue of which entity plays the trumpet in them. And this is simply to say that they are distinguished by the identity of some of the entities they involve). ${ }^{25}$

A broadly theistic case for the idea that haecceitistic differences must be ruled out is due to Leibniz. Leibniz had it that no merely possible world can differ haecceitistically from the actual one because, if some were to do so, then God would have made a choice between indifferent options when willing that a certain world be made actual. And in doing so-_Leibniz thought_-God would have ipso facto violated the Principle of Sufficient Reason (PSR):

[I]t is impossible there should be a reason why God, preserving the same situations of bodies among themselves, should have placed them in space after one certain particular manner and not otherwise-why everything was not placed the quite contrary way, for instance, by changing east into west. ${ }^{26}$

This famously led Leibniz to ban indiscernibles - among them, points in absolute space. ${ }^{27}$ For he had it that, were there indiscernibles, there would also be indiscernible possibilities for them, and hence indifferent options between which God would have made a choice when creating the actual world.

It is an indifferent thing to place three bodies, equal and perfectly alike, in any order whatsoever, and consequently they will never be placed in any order by him who does nothing without wisdom. But then, he being the author of

\footnotetext{
24 Thanks to an anonymous referee for the latter example and for pressing this point.

25 Incidentally, Adams was explicit in declaring that his form of Haecceitism did not commit him to any doctrine of haecceities. For the differences between a haecceity in the relevant sense and his notion of a thisness, see Adams (1981).

26 The passage is from Leibniz's third letter to Clarke. The present translation by Clarke is in Leibniz and Clarke: Correspondence, edited by Roger Ariew (2000): 15.

27 See Rodriguez-Pereyra (1999) for an excellent analysis.
} 
things, no such things will be produced by him at all, and consequently, there are no such things in nature. ${ }^{28}$

The options that-Leibniz says-God could not have chosen between with sufficient reason are options that differ haecceitistically. In the conjecture of God being able to change East into West, for instance, the world that would result from such a shifting would differ from the actual one merely as to which points of space are occupied by which entities. Similarly for the hypothesis of God having to arrange in some order-as opposed to others that are indiscernible from the one She does choosethree indiscernible bodies.

Leibniz is explicitly classified by Adams "as the archetypal believer in a purely qualitative universe" 29 -i.e., as taking the first horn of Adams's question. ${ }^{30}$

(This gives me the chance to further clarify the distinction between metaphysical (anti-)Haecceitism in my sense and "modal" (anti-)Haecceitism in Lewis's. The Lewisian anti-Haecceitist has it that no two worlds are qualitatively alike and yet differ in what they represent de re. ${ }^{31}$ A metaphysical anti-Haecceitist in my sense seeks to rule out what I call haecceitistic differences, where the latter notion is significantly different from the homonymous one that Lewis employs. In effect, the notion of a haecceitistic difference I work with does not rest on representation de re in any way. For a world $w$ whose denizens are worldbound would differ haecceitistically in my sense from the actual world $\alpha$ provided $w$ were qualitatively indiscernible from $\alpha$ even if $w$ were just like $\alpha$ in what it represents de re: the Leibnizian God could not have chosen to actualize $\alpha$ as opposed to $w$ without violating PSR. The Lewisian anti-Haecceitist, by contrast, has no reason to rule out that both $\alpha$ and $w$ exist. ${ }^{32}$

Importantly, when I say that metaphysical (anti-)Haecceitism is not modal (anti-) Haecceitism I do not mean that the former pair of doctrines do not have any modal entailments - to the contrary. It is nonetheless the case that a haecceitistic difference in my sense, as opposed to the homonymous phenomenon defined by Lewis, can arise - if at all-even within a single world. This is crucial for the broadly empiricist argument against haecceitistic differences-in my sense-that I shall now turn to. In what follows, I will mean by "haecceitistic difference" the notion I characterized above and leave the one employed by Lewis aside). ${ }^{33}$

A more recent argument against haecceitistic differences draws on certain features of modern physics. The argument is due, in the form I will consider, to Shamik Dasgupta. However, the case has its ancestry in other arguments that were discussed

28 The passage is from Leibniz's fourth letter to Clarke. The present translation by Clarke is in Leibniz and Clarke: Correspondence, edited by Roger Ariew (2000): 22.

29 Adams (1979): 5.

30 Ibid.: $\S$ II. I should mention that Adams's case to this end does not draw on the argument I presented: it draws on Leibniz's doctrine of complete individual concepts as stated in the Discourse on Metaphysics and in the letters to Arnauld instead. As far as the present topic is concerned, though, the outcome is the same: there cannot be indiscernible individuals-hence, no possible individual can give rise to haecceitistic differences (cf. Sect. 3 below).

31 See Lewis (1986): 221.

32 See ibid.: 224.

33 Thanks to two anonymous referees for making me clarify this point. 
at length in the philosophy of physics at least since Schrödinger and, more generally, in the wider context of a certain contention that has broadly empiricist roots.

Roughly, the idea is that facts of the matter that merely concern the identity of some entities, and haecceitistic differences in particular, lie beyond the limits of our knowledge and cannot contribute to the knowledge of the world we can acquire, either in general or in the light of our best physical theories. In line with this, Dasgupta views haecceitistic differences as danglers-i.e., as empirically undetectable and explanatorily redundant-in the light of physics from Newtonian Gravitational Theory (NGT) to this day.

Admittedly, Dasgupta does not explicitly formulate his case as one against 'haecceitistic differences'. His declared target is the claim that some facts are neither qualitative nor grounded in qualitative ones, and the things he explicitly claims to be danglers are what he calls 'primitive individuals' - roughly, the individuals those non-qualitative and non-qualitatively grounded facts are about.

That haecceitistic differences are exactly what his arguments do single out as danglers, though, can easily be appreciated by considering the examples he provides. ${ }^{34}$ In order to show that primitive individuals are explanatorily redundant, to start, Dasgupta asks us to consider "the following system": 35

[A] primitive individual called Peter is at an initial time $t_{0}$ propelled up in the air by a slingshot, only to fall by gravity back to Earth. And now consider a different system whose initial state at $t_{0}$ differs only in the fact that a different primitive individual, Paul, is slung. By hypothesis, we are to suppose that Peter and Paul have the same mass, shape, charge and so on. (...) [I]f the two systems both obey NGT, then Paul will make exactly the same trajectory through space as Peter. According to NGT, the identity of each particle makes no difference to how the slingshot or the Earth's gravitational field affect it. (...) [I]t is a straightforward consequence of NGT that mere differences in individualistic facts at an initial time do not give rise to differences in the future evolution of inter-particle distances or any other difference. ${ }^{36}$

To make the point that primitive individuals are empirically undetectable, Dasgupta says instead:

[I]magine a situation in which (...) a primitive individual is placed in front of you. Depending on what sorts of things primitive individuals are (or would be, if they were real!), this might be a situation in which you are in front of a chair,

\footnotetext{
${ }^{34}$ Dasgupta seems to think that there are haecceitistic differences only if there are primitive individuals in this sense. On top of this, he goes on to endorse the claim that no fundamental entity is an individual. While the latter claim does not strictly follow from his arguments against danglers, the idea seems to be that it does follow from those arguments along with the following tenets: (i.) only individuals are liable to give rise to haecceitistic differences; (ii.) the fundamental entities should not be liable to give rise to haecceitistic differences; (iii.) one should not hold that individuals respect PII-see Dasgupta (2009, 2014, 2017). The merit of (i.)-(iii.) is an issue that lies beyond the scope of this article. Thanks to an anonymous referee for asking me to mention this important point.

35 Dasgupta (2009): 41.

36 Ibid.
} 
or an electron, or perhaps something else. To fix ideas, let us suppose without loss of generality that it is a chair. And now imagine a situation in which everything is exactly the same except that a different primitive individual is in front of you. Suppose this different individual has exactly the same qualities as the actual chair in front of you: imagine it were colored the same, shaped the same, and so on. (...) [T] he situation would look and feel and smell exactly the same to you: we cannot tell the difference between situations that differ only in their individualistic facts. ${ }^{37}$

In both cases, mere differences in the identity of the entities involved in otherwise indiscernible situations-i.e., haecceitistic differences-are what is singled out as problematic in the light of NGT.

Dasgupta not only presented these arguments as supporting a view that takes all fundamental facts to be qualitative: he also mentioned Adams's aforementioned article in all of the three papers where he supported such a view, thus qualifying it as one that takes the first horn of Adams's question. ${ }^{38}$

Views that are classified as forms of anti-Haecceitism, I conclude, share a common rationale: the idea that haecceitistic differences are to be ruled out.

\section{Anti-Haecceitism Without Fundamentality}

I considered a characterization of Haecceitism and anti-Haecceitism that classifies as a view of the former sort any metaphysics that denies there to be fundamentality. I claimed that such a characterization is certainly wrong-headed if some metaphysics can be such that there is no fundamentality and that the rationale for anti-Haecceitism is satisfied-i.e., that haecceitistic differences cannot possibly arise. I shall now argue that such a metaphysics is viable. Here is an example.

Suppose, to start, that for something to be fundamental is for it to be ungrounded. For simplicity, let me assume for the sake of the example that grounding is a relation between facts - thus, that facts are what may be ungrounded (hence fundamental) or grounded (hence non-fundamental). ${ }^{39}$ Suppose, however, that no fact is ungrounded because a form of infinitism is true. To appreciate the point, consider an infinite chain of facts $F, F^{\prime}, F^{\prime}$,,$\ldots$, such that $F$ is grounded in $F^{\prime}, F^{\prime}$ is grounded in $F^{\prime}$, and so on ad infinitum. ${ }^{40}$ Suppose all the facts there are belong to some infinite, nonwell-founded chain of this sort. Suppose that every entity there is fulfills a strong

\footnotetext{
37 Ibid.: 42.

38 See Dasgupta (2009): fn. 22; (2014): 25-26; (2017): 7.

39 For endorsements of the idea that a fact is fundamental if and only if it is ungrounded, see e.g. Schaffer (2009), Rosen (2010), Audi (2012), Dixon (2016), Bennett (2017), Shumener (2017), and Leuenberger (2020).

40 By "grounded in", here, I mean "partially grounded in" in the sense of "either fully grounded in, or literally partially grounded in". See Fine (2012): 50. Given such a reading of "grounded in", if grounding (i.e., partial grounding) is irreflexive and transitive (as per orthodoxy), the claim that every fact belongs to an infinite chain of the sort described is equivalent to the claim that every fact is grounded. Thanks to Fabrice Correia.
} 
version of PII (strong PII, henceforth)—one that imposes that no two things can be qualitatively just alike. Finally, suppose that strong PII holds not only within each possible world, but trans-worldly as well, so that no two possible things exactly resemble one another. ${ }^{41}$

Now, strong PII is not precisely stated yet. The advocate of the metaphysics I just sketched should of course define which traits of a thing count as qualitative- thus clarifying over how narrow a class of features the second-order quantifier may range in a true statement of PII:

$$
\forall x \forall y(\forall \mathrm{P}(\mathrm{P} x \leftrightarrow \mathrm{P} y) \rightarrow x=y)
$$

While diverse accounts of the qualitative/non-qualitative distinction were set forth ${ }^{42}$ some cases are typically taken to be uncontroversial. For instance, being identical to Miles Davis and being in love with Elvis Presley are typically taken to be paradigmatic examples of non-qualitative features. Being blue and being an only child, by contrast, are typically seen as paradigmatically qualitative. The distinction I take to be relevant here is more precisely the one that tells apart what are sometimes called the 'pure' features from the 'impure' ones. ${ }^{43}$ Thus, the features of being identical to some individual and of being in love with a brown-eyed girl also count as qualitative under my approach because they are pure: it is not the case that for something to have one of them is for it to be somehow related to one or more entities in particular. The idea is that a qualitative feature $F$ can be such that for something $x$ to have $F$ is for $x$ to be somehow related to some entities or others, as long as $F$ does not consist in being somehow related specifically to some given entities in particular as opposed to all others. Here is one way to characterize the distinction I have in mind:

\footnotetext{
${ }^{41}$ The coherence of infinitism about grounding was defended both in metaphysics and in the philosophy of physics. See, among others, Morganti (2014, 2018), Tahko (2014), Bohn (2018), and Bliss (2019). Loss (2021) positively argues that, granted certain assumptions about grounding, there are no ungrounded facts. For concrete instances of infinitism, see Morganti (2015): 569-570; (2018): 260, Bohn (2018): Sect. 3. Morganti (2015), in particular, presents a physical model-Georgi (1989)'s-in which, prima facie, all particles satisfy strong PII.

${ }^{42}$ See, among others, Carnap (1947), Hempel and Oppenheim (1948), Goodman (1955), Adams (1979), Rosenkrantz (1979), Khamara (1988), Humberstone (1996), Rodriguez-Pereyra (2006), Cowling (2015), Hoffmann-Kolss (2019), Plate (2021).

${ }^{43}$ I mention this because some take there to be features that are pure and still should not be regarded as qualitative, instead. In particular, as remarked by Cowling (2015), some view "structural properties" like "being distinct from something" as non-qualitative as they "contribute nothing to the "qualitative character" of the world"; ibid.: § 4.6. Cf. Bricker (2006). And being distinct from something is, of course, a pure feature. But it is not clear to me that the relevant intuition concerning the 'qualitative character' of the world should be taken seriously when defining a technical notion such as the qualitative/non-qualitative distinction. More importantly, I do not think that being distinct from something should be viewed as non-qualitative when it comes to duly stating Strong PII. For, roughly, if two possible things differ in that one is distinct from something and the other is not, then they differ in further, non-controversially qualitative respects as well: one is, and the other is not, alone - the only denizen of its own world. Thus, it would be vain to view being distinct from something as non-qualitative for the sake of duly stating Strong PII.
} 
$P$ is a non-qualitative property $=_{\mathrm{df}}$ For some things $a_{1}, a_{2}, \ldots, a_{n}, n \geq 1$, and some $\mathrm{n}+1$-ary relation $\mathrm{R}$, the property $\lambda x P x$ is identical to the property $\lambda x$ $\mathrm{R}\left(x, a_{1}, \ldots, \mathrm{a}_{\mathrm{n}}\right)$.

Every property that is not non-qualitative is qualitative. ${ }^{44}$

I am not claiming that this characterization is flawless. The point here is rather that, under such a characterization of 'qualitative', things that satisfy strong PII cannot give rise to haecceitistic differences. For the characterization classifies a feature of being identical to a given entity in particular as non-qualitative. Thus, if Strong PII holds true of all possible things, then no two possible things differ merely as concerns their identity. And if so, then no two possible situations differ merely in the identity of some of the entities involved in them, respectively. Hence, there are no haecceitistic differences in the metaphysics I have been describing: every difference with respect to which entity is involved in given situations is necessarily accompanied by a difference over and above the identity of entities. ${ }^{45}$

The view I just sketched thus satisfies the desideratum set forth by the typical arguments for anti-Haecceitism. However, the characterizations I considered (in Sect. 1) classify it as a form of Haecceitism. ${ }^{46}$ Note that it would not do to modify those characterizations as follows:

ANTI-HAECCEITISM.

(i.) There is a fundamental level of reality, and:

(ii.) Such a fundamental level is qualitative.

\section{HAECCEITISM*}

(i.*) There is a fundamental level of reality, and:

(ii.**) Such a fundamental level is not qualitative.

\footnotetext{
${ }^{44}$ I consider a distinction that applies to properties for the sake of simplicity only. I do not mean to suggest in any way that the distinction cannot be effectively rephrased in nominalized terms.

45 Thanks to an anonymous referee for demanding clarifications on the qualitative/non-qualitative distinction.

46 An anonymous referee, whom I thank, drew my attention to the fact that this might not be the case under certain approaches that do not identify the fundamental with the ungrounded. An example is given in Raven (2016). Raven offers reasons for defending a non-foundation conception of fundamentality such that "an entity is fundamental (or ineliminable (...)) just in case not all facts about it are grounded in facts about other entities"; ibid.: 607. As such a conception is one where infinitist metaphysics can still include fundamental entities, the metaphysics I described in the main text is not misclassified as haecceitistic just because it includes no ungrounded entities. This might give one a conditional claim for a nonfoundational view of fundamentality such as Raven's: if one thinks that ANTI-HAECCEITISM is a good characterization of anti-Haecceitsm, then one should not take the fundamental to be the foundational. But there are other ways to defend a form of Anti-Haecceitism about the fundamental that I take to have the advantage of not committing one to any particular conception of the fundamental itself-cf. Sect. 4 below. Moreover, I submit that one might hold that there is no fundamentality in any sense and still be entitled to take a position in the debate between Haecceitism and anti-Haecceitism.
} 
For then the view I have described would turn out to be one where both Anti-Haecceitism and Haecceitism are false. But it should not: it should be recognized to be a view that the anti-Haecceitist—in the light of her own argument-has no reason to reject, and has reason to prefer, ceteris paribus, over others.

Nor would one properly solve the issue by characterizing both Haecceitism and anti-Haecceitism as disjunctive claims. The result would be the following:

ANTI-HAECCEITISM*.

(i.*) There is no fundamental level of reality, or:

(ii.*) The fundamental level of reality is qualitative.

\section{HAECCEITISM.}

(i.) There is no fundamental level of reality, or:

(ii.) The fundamental level of reality is not qualitative.

For then the infinitist metaphysics I described would be one in which both Haecceitism and anti-Haecceitism(*) are true. I submit that this would be an unpalatable result. It might be that the anti-Haecceitist has a way to rule out haecceitistic differences without committing to strong PII. But it is a metaphysics where every possible thing does respect strong PII I have described. And the idea that strong PII is at least possibly false has been the reason par excellence that Haecceitists have advanced against forms of anti-Haecceitism that entail its necessary truth. Moreover, it is at least desirable that Haecceitism and anti-Haecceitism be stated as mutually exclusive claims, while HAECCEITISM and ANTI-HAECCEITISM* are not mutually exclusive. Thus, I hold that we should look for characterizations that qualify the infinitist metaphysics above as one where Haecceitism is false. ${ }^{47}$

In the next section, I will introduce a proposal as to how Haecceitism and antiHaecceitism can be characterized without appealing to the notion of fundamentality. While not providing a conclusive argument in support of such characterizations, I will show that they rightly classify the infinitist metaphysics I depicted as a form of anti-Haecceitism-thus faring better than the definitions considered above.

\section{Anti-Haecceitism Rephrased}

My proposal is very simple. Instead of imposing on the anti-Haecceitist a claim in terms of fundamentality that she might-consistently with her own desideratum on an acceptable metaphysics - reject, I suggest that we characterize her view in terms of that very desideratum. As I said, such a desideratum consists in the idea that the possibility of haecceitistic differences is to be excluded. More precisely, depending on the argument that leads her to that idea, the anti-Haecceitist will hold that haecceitistic differences involving entities of a given category are to be ruled out. For

\footnotetext{
$\overline{47}$ Thanks to an anonymous referee for bringing the option of ANTI-HACCEITISM* to my attention.
} 
different arguments for anti-Haecceitism single out different entities as such that they should not be allowed to possibly give rise to haecceitistic differences. According to Leibniz's argument, the entities that cannot give rise to haecceitistic differences are all the entities that are, directly or indirectly, the objects of God's choice when She creates. According to arguments such as Dasgupta's, they are the entities that are subject to post-Newtonian physical laws.

I suggest that we take anti-Haecceitism about the entities of a certain categorythe $K \mathrm{~s}$ - as the doctrine that no $K$ is such that it can give rise to haecceitistic differences. That is:

ANTI-HAECCEITISM about the $K$ s.

No $K$ is such that it can give rise to haecceitistic differences.

HAECCEITISM about the $K$ s.

Some $K$ s are such that they can give rise to haecceitistic differences.

Leibniz's metaphysics will then count as a form of anti-Haecceitism about the entities that are objects of God's choice when creating - in particular, about worlds and about individuals. Those convinced by arguments such as Dasgupta's will accept a form of anti-Haecceitism about the entities that are subject to post-Newtonian physical laws. If so inclined, one might take the $K$ s to be the fundamental entities and defend a metaphysics that is anti-Haecceitistic about them. Thus, while it disentangles the commitments of anti-Haecceitism from concerns about fundamentality, my phrasing of the former is perfectly compatible with there being forms of anti-Haecceitism that make claims about the fundamental-in particular, about the fundamental entities.

And the anti-Haecceitist might take the $K$ s to be all the entities whatsoever. This would give her a way to endorse the tenet, which I considered in Sect. 1, that reality tout court is qualitative. None of the arguments for anti-Haecceitism discussed above, however, does support such a tenet.

My phrasing rightly classifies the infinitist metaphysics described in Sect. 3 as a form of anti-Haecceitism, as no possible entity such a metaphysics accepts can give rise to haecceitistic differences.

It can also account for an intuition that one might have concerning haecceitistic differences in different infinitist worlds. To see the point, consider a gunky world $w$, that is, a world $w$ such that every part of $w$ has proper parts. For the sake of simplicity, suppose one can 'partition' $w$ in terms of the mereological structure of the objects in it. Call the resulting partitions 'levels'. As with an informal counterpart of the set-theoretic partition of a given set, no distinct levels overlap, and the union of all the levels is $w$ itself.

Suppose that in each level there are only those proper parts of $w$ that belong to one and the same 'mereological level', so to speak. By way of illustration, say that one level includes only the atoms-in the chemical, not in the mereological, sense of the term-while another includes only the molecules, and still a further one only the mid-sized dry objects, and so on. (This is of course over-simplified, but it is only meant to provide a toy example). Given this construction, suppose we want to express the claim that parts are more fundamental than wholes. Here is a way that will prove to be extremely helpful in what follows. 
Say that a level $l_{n}$ is more fundamental than level $l_{m}$ iff, for every composite object $c$ in $l_{m}$, there is a proper part $p$ of $c$ in $l_{n}$. This defines a notion of relative fundamentality between levels. We then use this to define a notion of relative fundamentality between objects: object $o_{n}$ is more fundamental than object $o_{m}$ iff $o_{n}$ belongs to a level that is more fundamental than the one $o_{m}$ belongs to.

Now, consider a world $w^{*}$ that is mereologically just like $w$. For instance, if at level $l_{n}$ there are only atoms in $w$, at level $l_{n}{ }^{*}$ there are only atoms in $w^{*}$, and so on.

There are no mereological differences between $w$ and $w^{*}$. But suppose there are haecceitistic differences in $w$. Suppose, further, that the first level of $w$ that includes haecceitistic differences is level $l_{n}$-where: level $l_{n}$ is the first level at which $\varphi$, for a general formula $\varphi$, iff (i) at $l_{n}, \varphi$, and for every level $l_{m}$, if $\varphi$ holds at $l_{m}, l_{m}$ is less fundamental than $l_{n}$.w is such that if there are other heacceitistic differences in $w$ beside the ones at level $l_{n}$, they are all at levels that are less fundamental than $l_{n}$. By contrast, in $w^{*}$ we have that there are haecceitistic differences at level $l_{n}{ }^{*}$, but there are no haecceitistic differences at levels that are less fundamental than $l_{n}{ }^{*}$. The only other haecceitistic differences in $w^{*}$ are at levels $l_{o}^{*}$ that are more fundamental than $l_{n}{ }^{*}$ - this entails, in effect, that $l_{n}{ }^{*}$ is not the first level at which there are haecceitistic differences.

Then one might want to say that $w^{*}$ is more robustly haecceitistic than $w$, insofar as the levels in $w^{*}$ that include haecceitistic differences are more fundamental than the levels in $w$ that do (with the exception of $l_{n}$ and $l_{n}{ }^{*}$ ). Note that the comparison can be done even if both $w$ and $w^{*}$ are infinitist worlds. ${ }^{48}$

There are intuitions, by contrast, that do not sit well with my account of the present debate. Suppose, for instance, that individuals respect strong PII, and still they have haecceities in the sense discussed above (Sect. 2) - that for every individual $a$, there is a haecceity $h$ such that $a$ is the very individual it is by virtue of having $h$. Then one might want to count such a metaphysics as haecceitistic tout court despite the fact that, at least prima facie, no haecceitistic difference can arise in it. ${ }^{49}$

Yet under my approach to the debate, the metaphysics in question would count as a form of anti-Haecceitism about individuals. For, as I said in Sect. 3, things that respect strong PII cannot possibly give rise to haecceitistic differences. In effect, any difference with respects to which of them is involved in given situations must be accompanied by a further difference that is purely qualitative. For any two of them must differ in some purely qualitative respect themselves.

The metaphysics in question might however still be one that is haecceitistic about the haecceities themselves - for instance, if there is a level of reality at which only haecceities exist, quite independently from the individuals that instantiate them, and at that level the haecceities, which are qualitatively indiscernible from one another, are such that they can give rise to haecceitistic differences. ${ }^{50}$ If this is the case, and

\footnotetext{
${ }_{48}$ Thanks to an anonymous referee for suggesting this case. I am grateful to Claudio Calosi for discussion on this point.

49 Thanks to an anonymous referee for suggesting this case.

50 For endorsements of haecceities and of the idea that haecceities can exist without their bearers, see Plantinga (1976), Rosenkrantz (1993), Williamson (2013). The whole doctrine is explicitly rejected in Adams (1981).
} 
if haecceities are the only fundamental entities, then the metaphysics in question would of course be a form of Haecceitism about the fundamental entities. If to be a Haecceitist tout court is to be a Haecceitist about all entities, though, it does not count as a form of Haecceitism tout court, because it is a form of Anti-Haecceitism about individuals under the characterization I gave earlier in this section. ${ }^{51}$

For, to stress once more, entities that fulfil a strong form of PII are such that they cannot give rise to haecceitistic differences.

This points to an important consideration. That the entities of a given category respect strong PII is a sufficient condition for them to not possibly give rise to haecceitistic differences. Is it a necessary one, as well? In other words, is Adams right in thinking that the anti-Haecceitist about the $K \mathrm{~s}$ is committed to the claim that the $K \mathrm{~s}$ satisfy a strong version of PII? While I think that he is and that, as I mentioned, the typical rationale against anti-Haecceitism-hence, for Haecceitism-about the $K$ s is the idea that the $K$ s should not be taken to satisfy a strong version of PII, these are points that lie beyond the scope of the present paper.

Acknowledgements For their help and kindness in discussing previous versions of this paper, I thank Riccardo Baratella, Gaétan Bovey, Claudio Calosi, Fabrice Correia, Vincent Grandjean Perrenoud-Contesse, Marco Marabello, Robert Michels, Gonzalo Rodriguez-Pereyra, Thomas Sattig, Antoine Taillard, Lisa Vogt, Jonas Werner, Tobias Wilsch, and the participants to a Colloque de Recherche at the University of Neuchâtel, to a Tübingen Oberseminar in Theoretical Philosophy, and to the workshop "Things and Their Explanatory Power: Essence, Grounding, and Causation" at the University of Bern. I am also grateful to three anonymous referees for this journal. This work was generously funded by the Swiss National Science Foundation Early Postdoc.Mobility Grant P2NEP1_200132.

Funding Open access funding provided by University of Neuchâtel.

Open Access This article is licensed under a Creative Commons Attribution 4.0 International License, which permits use, sharing, adaptation, distribution and reproduction in any medium or format, as long as you give appropriate credit to the original author(s) and the source, provide a link to the Creative Commons licence, and indicate if changes were made. The images or other third party material in this article are included in the article's Creative Commons licence, unless indicated otherwise in a credit line to the material. If material is not included in the article's Creative Commons licence and your intended use is not permitted by statutory regulation or exceeds the permitted use, you will need to obtain permission directly from the copyright holder. To view a copy of this licence, visit http://creativecommons.org/licen ses/by/4.0/.

\section{References}

Adams, R. M. (1979). Primitive thisness and primitive identity. The Journal of Philosophy, 76(1), 5-26.

Adams, R. M. (1981). Actualism and thisness. Synthese, 49(1), 3-41.

Ariew, R. (ed.) (2000). Leibniz and Clarke: Correspondence. Hackett Publishing.

Audi, P. (2012). Grounding: Toward a theory of the in-virtue-of relation. The Journal of Philosophy, 109(12), $685-711$.

Babic, J., \& Cocco, L. (2020). A note on Dasgupta's generalism. Philosophical Studies, 177(8), 2153-2162.

Bacon, A. (2019). Is reality fundamentally qualitative? Philosophical Studies, 176(1), 259-295.

Bennett, K. (2011). Construction area (no hard hat required). Philosophical Studies, 154(1), 79-104.

Bennett, K. (2017). Making things up. Oxford University Press.

Black, M. (1952). The identity of indiscernibles. Mind, 61(242), 153-164.

$\overline{51}$ Thanks to an anonymous referee for having me clarify my take on this. 
Bliss, R. (2019). What work the fundamental? Erkenntnis, 84(2), 359-379.

Bohn, E. D. (2018). Indefinitely descending ground. In R. Bliss \& G. Priest (Eds.), Reality and its structure: Essays in fundamentality (pp. 167-181). Oxford University Press.

Carnap, R. (1947). On the application of inductive logic. Philosophy and Phenomenological Research, 8(1), 133-148.

Correia, F. (2010). Grounding and truth-functions. Logique Et Analyse, 53(211), 251-279.

Correia, F. (2016). On the logic of factual equivalence. Review of Symbolic Logic, 9(1), 103-122.

Cowling, S. (2016). Haecceitism. The Stanford Encyclopedia of Philosophy (Fall 2016 Edition), Edward N. Zalta (Ed.). https://plato.stanford.edu/archives/fall2016/entries/haecceitism/

Cowling, S. (2015). Non-qualitative properties. Erkenntnis, 80(2), 275-301.

Dasgupta, S. (2017). Can we do without fundamental individuals? Yes. In E. Barnes (Ed.) Current controversies in metaphysics (pp. 7-24). Routledge.

Dasgupta, S. (2009). Individuals. An essay in revisionary metaphysics. Philosophical Studies, 145(1), 35-67.

Dasgupta, S. (2014). On the plurality of grounds. Philosophers' Imprint, 14(20), 1-28.

Dixon, S. T. (2016). What is the well-foundedness of grounding? Mind, 125(498), 439-468.

Fine, K. (2012). Guide to ground. In Correia \& Schnieder (Eds.), Metaphysical grounding. Understanding the structure of reality (pp. 37-80). Cambridge University Press.

Fine, K. (2001). The question of realism. Philosophers' Imprint, 1, 1-30.

Fine, K. (2005). Modality and tense. Oxford University Press.

Georgi, H. (1989). Effective quantum field theories. In P. Davies (Ed.), The new physics (pp. 446-457). Cambridge University Press.

Goodman, N. (1955). Fact, fiction, and forecast. Harvard University Press.

Hempel, C. G., \& Oppenheim, P. (1948). Studies in the logic of explanation. Philosophy of Science, 15(2), $135-175$.

Hoffmann-Kolss, V. (2019). Defining qualitative properties. Erkenntnis, 84(5), 995-1010.

Humberstone, I. L. (1996). Intrinsic/extrinsic. Synthese, 108(2), 205-267.

Kaplan, D. (1975). How to Russell a Frege-Church. The Journal of Philosophy, 72, 716-729.

Khamara, E. J. (1988). Indiscernibles and the absolute theory of space and time. Studia Leibnitiana, 20(2), $140-159$.

Leuenberger, S. (2020). The fundamental: Ungrounded or all-grounding? Philosophical Studies, 177(9), 2647-2669.

Lewis, D. (1986). On the plurality of worlds. Blackwell.

Linnebo, Ø. (2014). 'Just is'-statements as generalized identities. Inquiry, 57, 466-482.

Loss, R. (2021). There are no fundamental facts. Analysis, 81, 32-39.

Morganti, M. (2014). Metaphysical infinitism and the regress of being. Metaphilosophy, 45(2), 232-244.

Morganti, M. (2015). Dependence, justification and explanation: Must reality be well-founded? Erkenntnis, $80(3), 555-572$.

Morganti, M. (2018). The structure of physical reality: Beyond foundationalism. In R. Bliss \& G. Priest (Eds.), Reality and its structure: Essays in fundamentalitys (pp. 254-272). Oxford University Pres.

Plantinga, A. (1976). Actualism and possible worlds. Theoria, 42, 139-160.

Plate, J. (2021). Qualitative properties and relations. Philosophical Studies, 20, 1-26.

Raven, M. (2016). Fundamentality without foundations. Philosophy and Phenomenological Research, 93(3), $607-626$.

Rodriguez-Pereyra, G. (1999). Leibniz's argument for the identity of indiscernibles in his correspondence with Clarke. Australasian Journal of Philosophy, 77(4), 429-438.

Rodriguez-Pereyra, G. (2006). How not to trivialize the identity of indiscernibles. In P. F. Strawson \& C. L. Chabrakarti (Eds.), Universals, concepts and qualities (pp. 205-223). Routledge.

Rosen, G. (2010). Metaphysical dependence: Grounding and reduction. In B. Hale \& A. Hoffmann (Eds.), Modality: Metaphysics, logic, and epistemology (pp. 109-136). Oxford University Press.

Rosenkrantz, G. S. (1979). The pure and the impure. Logique Et Analyse, 22, 515-523.

Rosenkrantz, G. S. (1993). Haecceity. An ontological essay. Kluwer Academic Publishers.

Russell, J. S. (2016). Qualitative grounds. Philosophical Perspectives, 30(1), 309-348.

Russell, J. S. (2018). Quality and quantifiers. Australasian Journal of Philosophy, 96(3), 562-577.

Schaffer, J. (2009). On what grounds what. In D. Manley, D. Chalmers, \& R. Wasserman (Eds.), Metametaphysics: New essays on the foundations of ontology (pp. 347-383). Oxford University Press.

Shumener, E. (2017). The metaphysics of identity: Is identity fundamental? Philosophy Compass, 121(1), $1-13$.

Sider, T. (2020). The tools of metaphysics and the metaphysics of science. Oxford University Press. 
Tahko, T. (2014). Boring infinite descent. Metaphilosophy, 45(2), 257-269.

Williamson, T. (2013). Modal logic as metaphysics. Oxford University Press.

Publisher's Note Springer Nature remains neutral with regard to jurisdictional claims in published maps and institutional affiliations. 